\title{
Brachial Artery Tear Occurring Concurrently with A Distal Biceps Injury Led to A Diagnosis of Ehlers-Danlos Syndrome Type IV
}

\author{
Gerardo Gonzalez ${ }^{1 *}$, Rajeev Dayal ${ }^{1,2}$, Lee Andy ${ }^{1,2}$ and Alexander Golant ${ }^{3,4}$ \\ ${ }^{1}$ Department of Vascular Surgery, New York Presbyterian/Queens Flushing, Queens, USA \\ ${ }^{2}$ Department of Clinical Surgery, Weill Medical College of Cornell University, USA \\ ${ }^{3}$ Department of Sports Medicine, Orthopaedics and Rehabilitation, Weill Medical College of Cornell University USA \\ ${ }^{4}$ Department of Clinical Orthopaedic Surgery, Weill Medical College of Cornell University, USA
}

*Corresponding author: Gerardo Gonzalez, Department of Vascular Surgery, New York Presbyterian/Queens Flushing, NY, Queens, USA, Tel: +1718-670-2000; Email: geg9039@nyp.org

Rec date: June 22, 2017; Acc date: July 21, 2017; Pub date: July 24, 2017

Copyright: $\odot 2017$ Gonzalez G, et al. This is an open-access article distributed under the terms of the creative commons attribution license, which permits unrestricted use, distribution, and reproduction in any medium, provided the original author and source are credited.

\begin{abstract}
Ehlers-Danlos Syndrome (EDS) type IV is a connective tissue disorder with autosomal dominant inheritance. It can be potentially life-threatening due to increased risk of arterial rupture. The diagnosis is based on clinical findings including thin, translucent skin; bleeding propensity, rupture of vessels, and viscera. Isolated peripheral vascular injury may be the first presenting complaint, leading to eventual diagnosis in patients who often experience minimal trauma. We present a case of a brachial artery rupture requiring surgical reconstruction, eventually leading to the diagnosis of EDS type IV after a non-contact, low-energy injury to the arm of an adolescent.
\end{abstract}

Keywords: Brachial artery; Distal biceps injury; Ehlers-Danlos syndrome; Translucent skin; Antecubital fossa

\section{Introduction}

The vascular type of Ehlers-Danlos Syndrome (EDS) is characterized by thin, translucent skin; visceral rupture, and vascular fragility. Skin hyperextensibility and joint hypermobility are not hallmarks of this subtype of EDS. Isolated biceps tendon ruptures are rare injuries, especially in adolescents, and major vascular injuries have not been described in association with typical biceps tendon ruptures occurring from low-energy trauma [1]. Patients with connective tissue disorder may be predisposed to vascular injury even in the absence of significant trauma, and this is particularly the case in patients with Ehlers Danlos Syndrome (EDS) type IV. We present a case of a brachial artery rupture in a teenage boy from a non-contact, low-energy injury, and describe how the suspicious nature of this injury led to further evaluation and diagnosis of EDS type IV. The patient and family consented to publication of this report [2-5].

\section{Case Presentation}

A 16-year-old boy with no previous known medical history presented to our emergency room complaining of pain and swelling in the antecubital fossa of his right elbow which developed suddenly while playing handball. He reported feeling sudden onset of sharp pain as he hit the ball with his hand, but denied elbow hyperextension. Physical examination showed a large hematoma and ecchymosis in the anterior aspect of the antecubital fossa, the distal arm, and in the antecubital fossa; limited motion of the elbow, a well-perfused hand, and normal neurologic exam (Figure 1).

Plain radiographs of the elbow were negative. Patient was discharged with a sling, but he then returned to the emergency room two days later complaining of persistent pain. Physical examination showed large tense swelling in the antecubital fossa with ecchymosis. The anterior compartment of the arm was firm but compressible and there was no evidence of a compartment syndrome [6]. Radial and ulnar pulses were normal, but the brachial pulse was not palpable due to the hematoma.

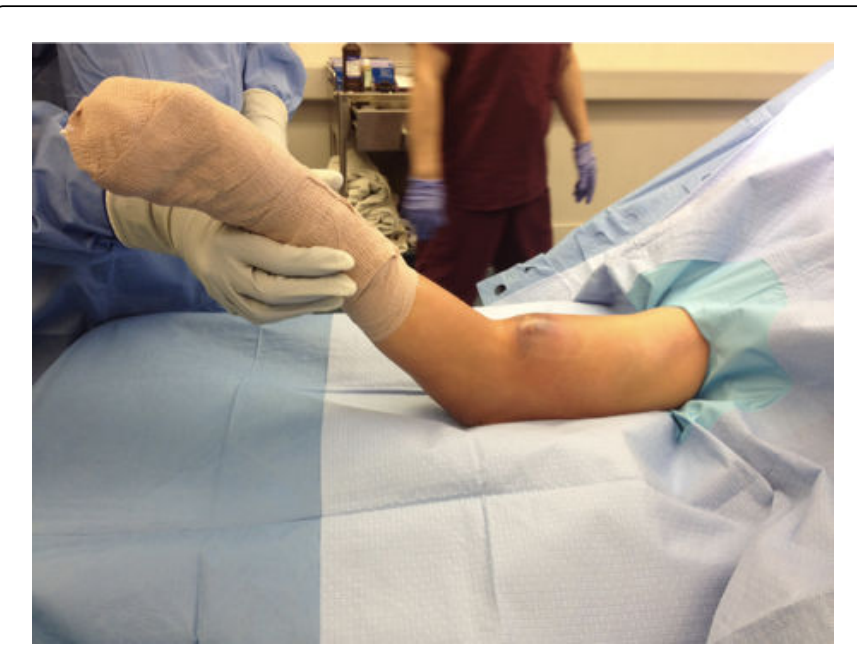

Figure 1: Preoperative photograph of the patient's arm, showing large tense swelling and ecchymosis just proximal to the antecubital fossa-medial view.

Several stigmata of connective tissue disorder were also noted on exam, including joint hyperlaxity. Extensive questioning of the patient and his parents revealed a history of easy bruising, but no other notable factors. MRI of the elbow was obtained the same day (Figure 2 ), and showed a partial tear at the distal biceps musculotendinous junction with a large hematoma. Coagulation studies were within 
normal limits. Due to persistent pain and to improve long-term function, the patient was taken to the operating room for evacuation of the hematoma and repair of the distal biceps with the orthopedics service.

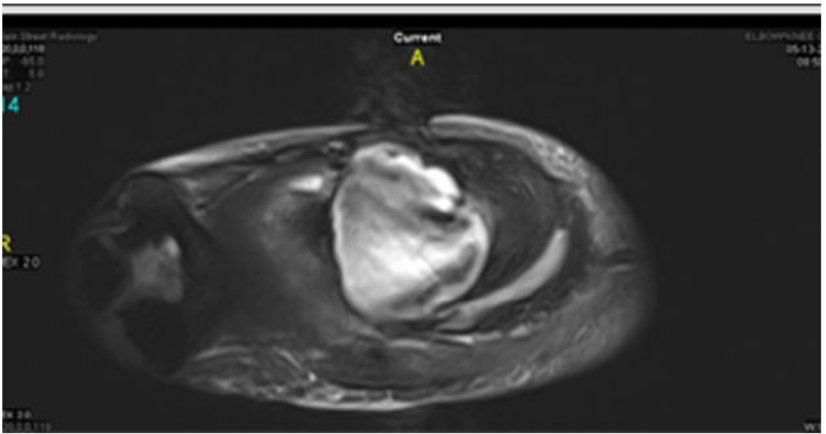

Figure 2: Preoperative MRI of the patient's elbow. Axial view proximal to the elbow, showing the hematoma deep to the biceps muscle belly.

\section{Procedure}

Surgery was performed under general anesthesia. A curvilinear incision was used, extending from the lateral border of the biceps medially across the elbow joint and distally continuing as the Henry volar approach to the forearm. After incising the fascia over the anterior compartment of the arm, a large amount of hematoma was encountered. Upon hematoma evacuation, there was obvious arterial bleeding from the medial aspect of the arm. After failing to control this bleeding directly, a tourniquet was inflated and vascular surgery consultation was called.

Vascular exploration revealed a complex tear of the brachial artery along approximately $2 \mathrm{~cm}$ of its length at the same level as the biceps muscle/tendon injury. Dissection was performed proximally and distally to mobilize the artery, and it was determined that direct repair was not possible due to the length of the injury. Due to the fragility of the artery, tourniquet was used to maintain control rather than direct control of the vessel. An autogenous cephalic vein graft was then harvested from the ipsilateral forearm and was used to perform an interposition graft. Pledgeted polypropylene sutures were used, as the artery was extremely fragile (Figure 3). After deflating the tourniquet, excellent flow through the brachial artery and the graft was observed. Total tourniquet time was 85 minutes. Distal pulses were also noted to be intact with direct palpation and Doppler ultrasound.

The distal biceps tear was then repaired directly with two 1 braided sutures. Forearm fasciotomy was not performed as the extremity was not ischemic for a prolonged period of time. A closed-suction drain (Jackson-Pratt) was placed, the wound was closed in layers, and a wellpadded posterior long-arm plaster splint was applied with the arm in 90 degrees of flexion.

Post-operative recovery was uneventful, and he maintained excellent perfusion of the forearm and hand with intact pulses. As bicep tendon ruptures are rare in adolescents, and this was associated with a significant vascular injury, there was concern that the underlying etiology was a connective tissue disorder. Therefore, during his post-operative recovery period the patient was referred for multidisciplinary evaluation to evaluate for connective tissue disorders.

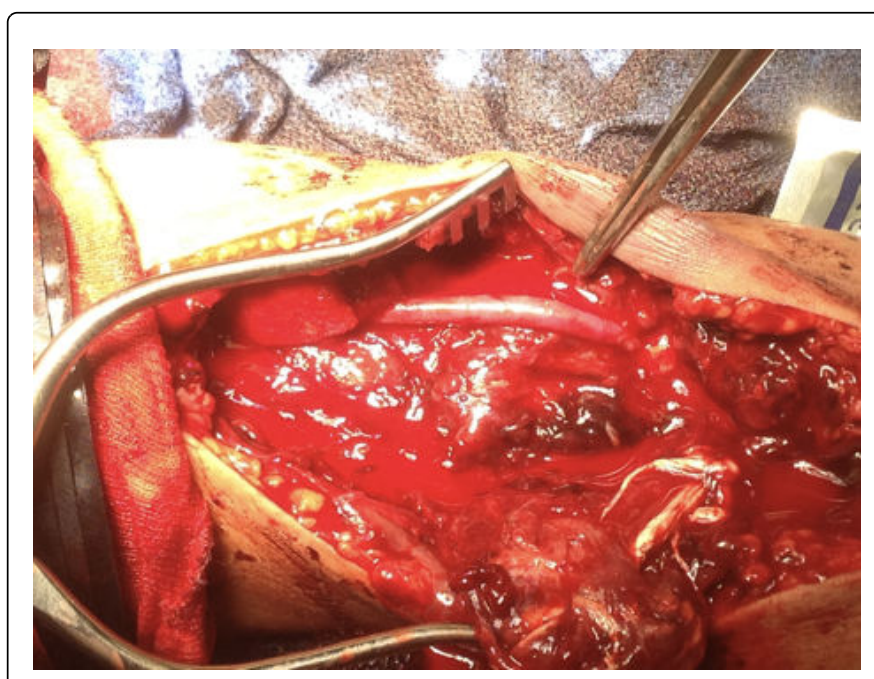

Figure 3: Intraoperative photograph of the interpositional graft with the cephalic vein, used to reconstruct the brachial artery rupture.

Evaluation included genetic testing for a panel of genes associated with connective tissue diseases associated with aortopathy and vascular dissections including Marfan syndrome, Loeys -Dietz, and Ehlers Danlos syndrome (ACTA2, CBS, COL3A1, COL5A1, COL5A2, FBN1, FBN2, MYH11, SLC2A10, SMAD3, TGFBR1, TGFBR2) and he was diagnosed with Ehlers-Danlos Syndrome Type IV with a c. 3500_3501delGT ins AA mutation in the COL3A1. Further genetic testing is currently being performed on his first-degree relatives, and counseling is being given regarding future participation in sports and other strenuous activities. Magnetic resonance angiography (MRA) performed in the post-operative period has not revealed other arteriopathies. The bypass graft is being followed with duplex ultrasound surveillance every 6 months.

\section{Discussion}

Peripheral arterial involvement as a major presenting problem has been reported in a number of patients with Ehlers-Danlos Syndrome type IV [7-13]. In one of the case reports, the peripheral arterial injury was the "tip off" that led to the diagnosis of EDS type IV [7]. Prompt treatment of vascular injuries must be undertaken, as related complications including compartment syndrome and nerve palsies, and amputation for ischemic complications have been reported $[8,11]$. The presence of a distal biceps tendon tear after trauma is rare, and represents only $3 \%$ of all biceps tendon injuries [1]. They occur mostly commonly in men in the fifth or sixth decade of life, are exceedingly rare in women, and have not been reported in children. Despite the abundance of vascular and neurologic structures in close proximity to the distal biceps tendon, extensive search of the English language medical literature by the authors failed to find any reports of associated vascular injury in conjunction with distal biceps injuries. Therefore, the highly unusual presentation in this patient prompted a work-up for a connective tissue disorder that could predispose him to vascular injury.

Ehlers-Danlos syndrome (EDS) is a group of connective tissue disorders caused by a variety of genetic mutations. Tissue biopsies of 
patients with this disorder have shown abnormal ultrastructural properties of collagen and elastin [2]. While type III EDS has been reported to be the most debilitating form with respect to musculoskeletal function, [3] type IV EDS, known as the vascular type, is considered overall the most serious type of EDS, and is associated with decreased median life expectancy ( 45 years to 50 years), mainly due the risk of spontaneous arterial or organ rupture [4-14]. Given that arterial rupture can occur repeatedly in patients with EDS, careful lifelong follow-up is necessary [14].

The major clinical diagnostic criteria include arterial/intestinal fragility or rupture, uterine rupture, or family history of EDS [4]. Genetic abnormality has been found involving the gene COL3A1, on the chromosome locus 2q32.2, affecting type III collagen. Genetic testing can easily be accomplished with DNA based sequencing. Imaging studies of the vascular system in patients with type IV EDS may reveal arterial aneurysms and dissections. It is important to note that the classical manifestations of connective tissue disorders, such as joint hypermobility or skin hyperelasticity, may or may not be present in patients with Ehlers-Danlos syndrome type IV, [5,6] and proper work-up, including by a geneticist, must be performed even in their absence, if the disease is suspected.

A total body magnetic resonance angiogram (MRA) is recommended as a baseline study once diagnosis of EDS type IV is made. Aneurysms and pseudoaneurysms found on screening MRA may be treated prophylactically, to prevent life- or limb-threatening bleeding which can occur if these rupture [15].

\section{Conclusion}

We describe an unusual case of a boy with Ehlers-Danlos syndrome type IV, of a brachial artery rupture occurring together with a partial tear of the distal biceps from a low-energy force applied to the hand. The injury likely occurred to a previously compromised artery, possibly with a preexisting aneurysm that ruptured from low-energy trauma. This case highlights the importance of careful examination and timely recognition of vascular injuries in patients with connective tissue disorders, even after minor trauma.

\section{References}

1. Vidal AF, Drakos MC, Allen AA (2004) Biceps tendon and triceps tendon injuries. Clin Sports Med 23: 707-722.
2. Kobayasi $\mathrm{T}$ (2006) Dermal elastic fibres in the inherited hypermobile disorders. J Dermatol Sci 41: 175-185.

3. Stanitski DF, Nadjarian R, Stanitski CL, Bawle E, Tsipouras P (2000) Orthopaedic manifestations of Ehlers-Danlos syndrome. Clin Orthop Relat Res 213-221.

4. Germain DP, Herrera-Guzman Y (2004) Vascular Ehlers-Danlos syndrome. Ann Genet 47: 1-9.

5. Bellenot F, Boisgard S, Kantelip B, Maillard P, Tissandier P, et al. (1990) Type IV Ehlers-Danlos syndrome with isolated arterial involvement. Ann Vasc Surg 4: 15-19.

6. Sheiner NM, Miller N, Lachance C (1985) Arterial complications of Ehlers-Danlos syndrome. J Cardiovasc Surg (Torino) 26: 291-296.

7. Arici V, Corbetta R, Fossati LG, Odero A (2013) Acute first onset of Ehlers-Danlos syndrome type 4 with spontaneous rupture of posterior tibial artery pseudoaneurysm. Vascular 21: 43-45.

8. Bowers WH, Spencer JB, McDevitt NB (1976) Brachial-artery rupture in Ehlers-Danlos syndrome: an unusual cause of high median-nerve palsy: A case report. J Bone Joint Surg Am 58: 1025-1026.

9. Curley SA, Osler T, Demarest GB (1988) Traumatic disruption of the subclavian artery and brachial plexus in a patient with Ehlers-Danlos syndrome. Ann Emerg Med 17: 850-852.

10. Ikeda K, Osamura N, Kasashima S (2012) The bilateral ulnar artery involvement in Type IV Ehlers-Danlos syndrom- A case report. Hand Surg 17: 213-216.

11. Mankad K, Puppala S (2011) Severe vascular complication after an arm stretch in a child with Ehlers-Danlos syndrome. J Clin Rheumatol 17: 223.

12. Witz M, Lehmann JM (1997) Aneurysmal arterial disease in a patient with Ehlers-Danlos syndrome. Case report and literature review. J Cardiovasc Surg (Torino) 38: 161-163.

13. Sayin AG, Bozkurt AK, Cangel U, Köksal C, Oz B (2001) A brachial aneurysm in childhood caused by Ehlers-Danlos syndrome. J Cardiovasc Surg (Torino) 42: 687-689.

14. Nakanishi K, Tajiri N, Nakai M, Shimizu S (2014) Recurrent arterial aneurysm rupture of the upper extremity in a patient with vascular-type Ehlers-Danlos syndrome. Interact CardioVasc Thorac Surg 19: 702-704.

15. Gok E, Goksel OS, Alpagut U, Dayioglu E (2012) Spontaneous brachial pseudo-aneurysm in a 12-year-old with kyphoscoliosis-type EhlersDanlos Syndrome. Eur J Vasc Endovasc Surg 44: 482-484. 